\title{
Should we continue to isolate patients with vancomycin-resistant enterococci in hospitals?
}

\section{The routine use of contact precautions for patients with vancomycin-resistant enterococci cannot be justified once colonisation with this multidrug-resistant bacterium becomes endemic}

nfections with vancomycin-resistant enterococci (VRE), which have become more common in Australian hospitals since the late 1990s, are associated with poor patient outcomes. Patients with gastrointestinal colonisation of VRE are at greater risk of infection, and patients infected with VRE are at higher risk of all-cause mortality. ${ }^{1}$

During outbreaks, VRE is assumed to spread between patients mainly via the hands of health care workers or in the hospital environment. Widely recommended strategies for minimising the risk of VRE transmission include screening to identify colonised patients, and subsequent contact precautions to minimise crosstransmission. Many hospitals use contact precautions for patients colonised or infected with VRE on current and each subsequent hospital admission, assuming VRE colonisation is lifelong. These recommendations for contact precautions are based on observational studies conducted primarily during outbreaks, inductive reasoning based on the known transmission potential, and expert opinion. However, dissent has been expressed against the routine use of contact precautions, particularly in hospitals where VRE is endemic. $^{2}$

\section{"Universal interventions ... are likely to be more effective in preventing transmission in high-risk settings"}

VRE is endemic in many Australian hospitals. ${ }^{3} \mathrm{We}$ have recently changed our policy requiring the routine use of contact precautions for patients found to be colonised with VRE, to a risk-based policy applied to all patients at Alfred Health. By outlining the rationale for this change, we hope that it will inform VRE control policies at other Australian hospitals.

By comparing routine passive surveillance with a point prevalence survey, we found that a strategy of screening of close contacts of patients with VRE did not identify the majority of VRE carriers in hospital. ${ }^{4}$ This may be due to exposure to antibiotics having a major role in VRE acquisition in the endemic hospital setting. Consistent with other studies,

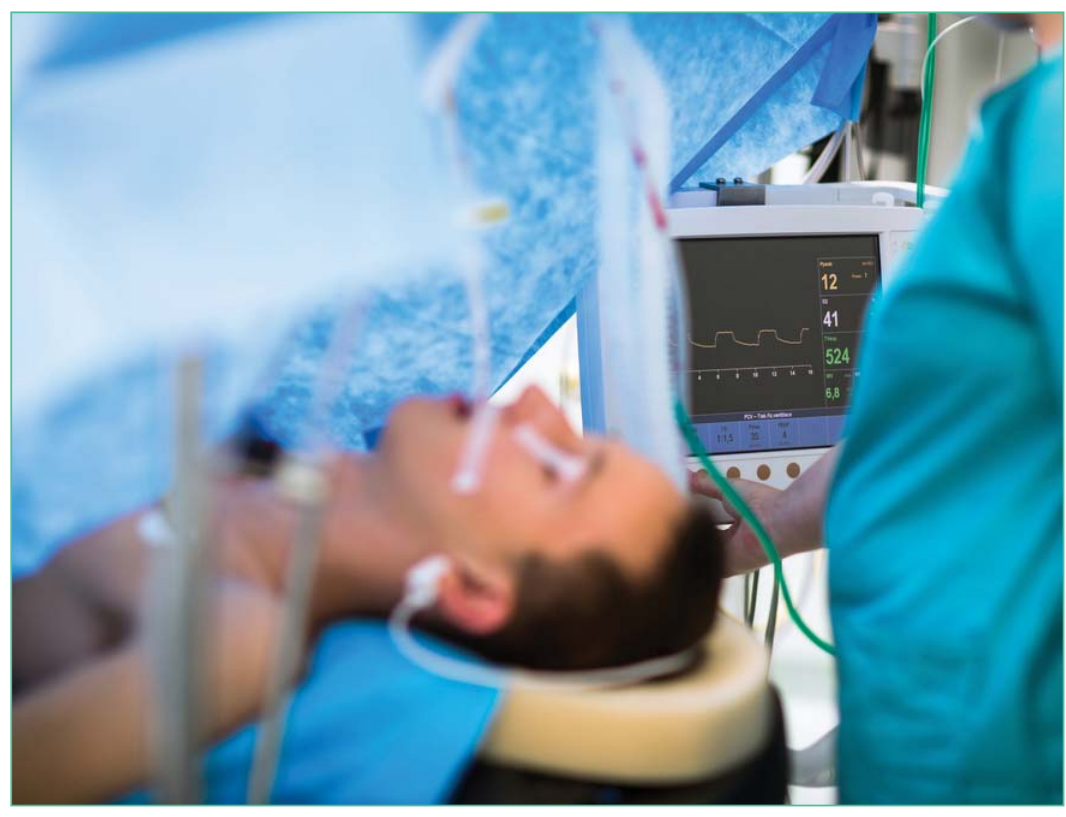

we have recently shown that antibiotic exposure, particularly to meropenem, is an important risk factor for VRE colonisation among patients. ${ }^{4}$ Although the magnitude of the effect of re-exposure to antibiotics on detectability and transmissibility of VRE has not been definitively established, we note that no patients who had colonisation detected more than 4 years prior were found to have VRE, despite $40 \%$ being exposed to antibiotics within the previous 3 months. ${ }^{5}$

In an earlier study where VRE transmission through contacts was documented, exposure to broadspectrum antibiotics was an important risk factor among incident cases. ${ }^{6}$ Therefore, these studies suggest that during cross-transmission of VRE in hospital, antibiotics are the major facilitator and predictor of new VRE acquisition. Similarly, a recent

Surendra Karki MSc, MSCIH, PhD ${ }^{1,2}$

Karin Leder FRACP, PhD, MPH ${ }^{1,3}$

Allen C Cheng FRACP, MPH, PhD

1 Monash University, Melbourne, VIC.

2 University of New South Wales, Sydney, NSW (current affiliation).

3 Royal Melbourne Hospital, Melbourne, VIC.

allen.cheng@ monash.edu

doi: 10.5694/mjal4.00672 study based on phylogenetic analysis and mapping of the vanB gene suggested that about half of hospitalacquired vancomycin-resistant Enterococcus faecium had recently acquired a transposon coding for vancomycin resistance. ${ }^{7}$ This sequence was the same as a $\operatorname{Tn} 1549$ sequence present in anaerobic bacteria, but was inserted in different sites in the E. faecium genome, suggesting that a substantial proportion of new VRE may have emerged through de-novo generation due to antibiotic selection pressure rather than cross-transmission. ${ }^{7}$

Studies also suggest that most patients clear detectable levels of VRE carriage in a relatively short period. ${ }^{5,8}$ Hospitals have varying policies by which patients are defined as cleared, based on screening of 
rectal swabs or faecal culture. Although a negative culture may not necessarily prove clearance, as intermittent shedding has been described, it is likely that a VRE-negative culture from a faecal specimen indicates either complete clearance or at least a very low density of VRE, which may have only marginal clinical significance. Recently, we studied the longterm carriage of VRE in a retrospective cohort study, and observed that only $12.6 \%$ of patients were positive for VRE if the initial detection was between 1 and 4 years before follow-up sampling, and none were positive if the initial detection was more than 4 years before follow-up. ${ }^{5}$ In addition, molecular typing suggested that at least half of the patients who remained VRE-positive at the time of the study were recolonised with new strains. ${ }^{5}$

Although contact precautions have been shown to minimise the risk of cross-transmission of VRE during outbreaks, there is accumulating evidence that they adversely affect the care of patients and impair patient flow. Studies, mostly conducted in hospitals in the United States, have found contact precautions are associated with adverse impacts on psychological outcomes, poorer satisfaction with care and perception of quality of care, less timely patient management, and fewer visits by health care workers. ${ }^{9}$ In studies conducted at our hospital, we have also found increased rates of non-pressurerelated injuries and medication errors, and delayed access to radiological investigations among patients colonised with VRE. ${ }^{10,11}$ While these impacts may be justified and mitigated where there are few colonised patients or in an acute outbreak setting, they are less justified in an ongoing endemic setting. This is particularly true for VRE, where subsequent clinically significant bloodstream infection is uncommon among colonised patients. ${ }^{12}$

What are the alternatives to contact precautions? Recent studies have shown that interventions that are universally applied (termed "horizontal" interventions) are more effective than those that are targeted to specific pathogens ("vertical" interventions, including contact isolation of patients colonised with VRE) in controlling multidrugresistant organisms. ${ }^{13}$ In a systematic review, we found that the universal daily topical application of $2 \%$ chlorhexidine gluconate using impregnated washcloths was associated with a reduction in new VRE colonisation, and also reduced methicillinresistant Staphylococcus aureus colonisation and central line-associated bloodstream infections. ${ }^{14}$ Thus, the use of chlorhexidine washcloths provides an example of a universal intervention not directed towards a specific pathogen, but rather having an impact on a wider range of important multidrugresistant organisms.

Similarly, effective antimicrobial stewardship programs should be another area of focus, as antibiotic selection pressure appears to be a significant factor associated with both emergence and spread of VRE in hospitals. In addition, elements of standard care such as adherence to hand hygiene, cleaning and disinfection after room separation and during room occupancy, hospital design elements including provision of sufficient toilets and bathrooms, and cleanable furnishings should be improved to reduce the risk of potential transmission of any multidrug-resistant organism in hospital. Furthermore, continued surveillance and review of hospital infection rates in high-risk areas are required to monitor for changes in epidemiology.

In conclusion, emerging evidence suggests that a significant proportion of VRE colonisation is attributable to exposure to broad-spectrum antibiotics; however, the clearance of carriage appears to be the rule, rather than the exception. Both these factors imply that only broad-based, continuous surveillance can identify patients with VRE.

If patients with VRE cannot easily be identified with faecal screening, then universal interventions, such as daily topical application of $2 \%$ chlorhexidine gluconate using washcloths, are likely to be more effective in preventing transmission in high-risk settings, such as intensive care units. Although the evidence supporting its use outside of intensive care units is weaker, we have found it to be feasible to provide washcloths to patients to self-apply after routine bathing in other high-risk settings such as haematology-oncology units. ${ }^{15}$ However, supervision and adherence may be a problem outside intensive care settings. Topical application of chlorhexidine gluconate using washcloths is also likely to reduce other significant infections, such as central line-associated bloodstream infections. A focus on horizontal rather than vertical interventions also avoids the adverse consequences associated with contact precautions. Limited facilities for isolating patients might then be better allocated to other hospital threats, such as norovirus or other multidrug-resistant pathogens.

Acknowledgements: We thank the staff of the Infection Prevention and Healthcare Epidemiology Unit at Alfred Health for their support and input.

Competing interests: No relevant disclosures.

Provenance: Not commissioned; externally peer reviewed.

References are available online at www.mja.com.au. 
1 Matar MJ, Tarrand J, Raad I, Rolston KV. Colonization and infection with vancomycin-resistant Enterococcus among patients with cancer. Am J Infect Control 2006; 34: 534-536.

2 Gardam M, Lemieux C, Evans G, et al. Response to the Provincial Infectious Diseases Advisory Committee Review of literature for evidence-based best practices for VRE control. 22 Oct 2012. http://www.sjhc.london.on.ca/sites/default/ files/pdf/VRE_response.pdf (accessed Mar 2014).

3 Coombs GW, Pearson JC, Daley DA, et al. Molecular epidemiology of enterococcal bacteremia in Australia. J Clin Microbiol 2014; 52: 897-905.

4 Karki S, Houston L, Land G, et al. Prevalence and risk factors for VRE colonisation in a tertiary hospital in Melbourne, Australia: a cross sectional study. Antimicrob Resist Infect Control 2012; 1: 31.

5 Karki S, Land G, Aitchison S, et al. Long-term carriage of vancomycin-resistant enterococci in patients discharged from hospitals: a 12-year retrospective cohort study. J Clin Microbiol 2013; 51: 3374-3379.

6 Zhou Q, Moore C, Eden S, et al; Mount Sinai Hospital Infection Control Team. Factors associated with acquisition of vancomycin-resistant enterococci (VRE) in roommate contacts of patients colonized or infected with VRE in a tertiary care hospital. Infect Control Hosp Epidemiol 2008; 29: 398-403.

7 Howden BP, Holt KE, Lam MM, et al. Genomic insights to control the emergence of vancomycin-resistant enterococci. MBio 2013; 4: e00412-e00413.

8 Sohn KM, Peck KR, Joo EJ, et al. Duration of colonization and risk factors for prolonged carriage of vancomycin-resistant enterococci after discharge from the hospital. Int J Infect Dis 2013; 17: e240-e246.

9 Stelfox HT, Bates DW, Redelmeier DA. Safety of patients isolated for infection control. JAMA 2003; 290: 1899-1905.

10 Karki S, Leder K, Cheng AC. Patients under contact precautions have an increased risk of injuries and medication errors: a retrospective cohort study. Infect Control Hosp Epidemiol 2013; 34: 1118-1120.

1 Karki S, Leder K, Cheng AC. Delays in accessing radiology in patients under contact precautions because of colonization with vancomycin-resistant enterococci. Am J Infect Control 2013; 41: 1141-1142.

12 Datta R, Huang SS. Risk of postdischarge infection with vancomycin-resistant enterococcus after initial infection or colonization. Infect Control Hosp Epidemiol 2010; 31: 1290-1293.

13 Huang SS, Septimus E, Kleinman K, et al. Targeted versus universal decolonization to prevent ICU infection. N Engl J Med 2013; 368: 2255-2265.

14 Karki S, Cheng AC. Impact of non-rinse skin cleansing with chlorhexidine gluconate on prevention of healthcareassociated infections and colonization with multi-resistant organisms: a systematic review. J Hosp Infect 2012; 82: 71-84.

15 Bass P, Karki S, Rhodes D, et al. Impact of chlorhexidineimpregnated washcloths on reducing incidence of vancomycin-resistant enterococci colonization in hematology-oncology patients. Am J Infect Control 2013; 41: 345-348 